\title{
Environmental fate and effects of organophosphate flame retardants in the soil-plant system
}

\author{
Qing Zhang ${ }^{1,2,4,}$, Weiping $\mathrm{Mei}^{3{ }^{3},}$, Longfei Jiang ${ }^{1}$, Qian Zheng ${ }^{3}$, Chunling Luo ${ }^{1,2,3,{ }^{*}, \text { Gan Zhang }}{ }^{1,2}$ \\ 1 State Key Laboratory of Organic Geochemistry and Guangdong-Hong Kong-Macao Joint Laboratory for Environmental Pollution and \\ Control, Guangzhou Institute of Geochemistry, Chinese Academy of Sciences, Guangzhou 510640, China \\ 2 CAS Center for Excellence in Deep Earth Science, Guangzhou 510640 China \\ 3 College of Natural Resources and Environment, South China Agricultural University, Guangzhou 510642, China \\ 4 University of the Chinese Academy of Sciences, Beijing 100049, China
}

H I G H L I G H T S

- Mechanisms of soil sorption and plant uptake of OPFRs were measured.

- Humid acids contribute to electrostatic interaction, hydrogen bonding, etc.

- Hydrolysis is an important transformation behavior of OPFRs in the soil-plant system.

- RCF showed no significant correlation with hydrophobicity of OPFRs in soil experiments.

\section{ARTICLE INFO}

Article history:

Received December 10, 2020

Revised February 15, 2021

Accepted February 17, 2021

Keywords:

OPFRs

Bioavailability

Transformation

Uptake

Translocation

\section{GRAPHICAL ABSTRACT}

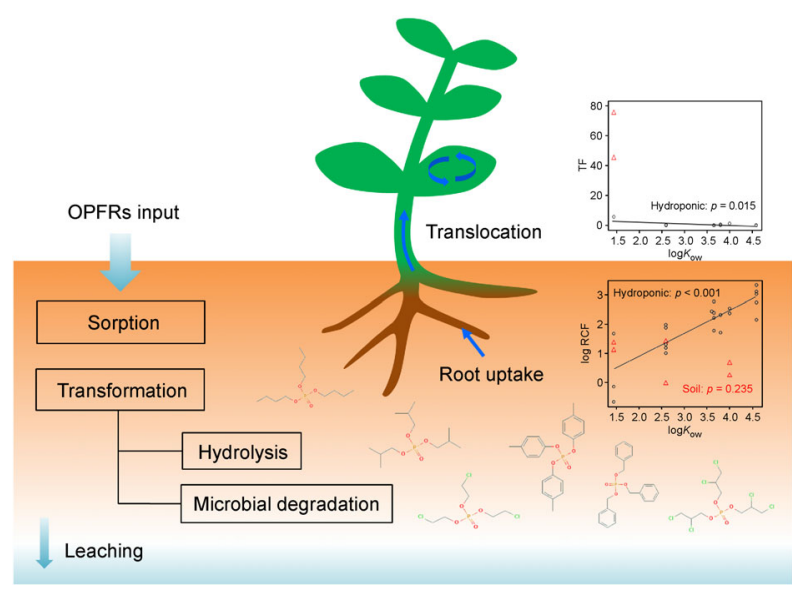

A B S TR A C T

Organophosphate flame retardants (OPFRs), as a replacement for polybrominated diphenyl ethers (PBDEs), are of increasing concern due to their high production over the years. Soil is the major environmental reservoir and interchange for OPFRs. OPFRs in soil could be transferred to the food chain, and pose potential ecological and human health risks. This review focused on the environmental fate and effects of typical OPFRs in the soil-plant system. We concluded that the sorption and transformation behaviors of OPFRs due to their crucial impact on bioavailability. The root uptake and translocation of OPFRs by plants were summarized with analyses of their potential affecting factors. The in planta transformation and potential ecological effects of OPFRs were also briefly discussed. Finally, we highlighted several research gaps and provided suggestions for future research, including the development of simulative/computative methods to evaluate the bioavailability of OPFRs, the effects of root exudates and rhizosphere microorganisms on the bioavailability and plant uptake of OPFRs, and the development of green and sustainable technologies for in situ remediation of OPFRs-contaminated soil.

(c) Higher Education Press 2021

\footnotetext{
* Corresponding author

E-mail address: clluo@gig.ac.cn (C. Luo)
}

'Qing Zhang and Weiping Mei contributed equally to this work. 


\section{Introduction}

Organophosphate esters are organic ester derivatives of phosphorous, used as insecticides, herbicides, nerve agents, and flame retardants for more than 150 years (van der Veen and de Boer, 2012). Organophosphate ester flame retardants (OPFRs), as a replacement for polybrominated diphenyl ethers (PBDEs), are of increasing concern due to their high production over the years. The global consumption of phosphorus-based flame retardants continuously increased from $11 \%$ to over $30 \%$ of flame retardant's total consumption from 2008 to 2017 . That of bromine-based flame retardants decreased from $23 \%$ to $19 \%$ (Wang et al., 2020b). Besides, OPFRs have been detected in various environmental matrices, such as outdoor air, indoor air and dust, water, soil, sediment, and biological matrices (Wei et al., 2015; Chen et al., 2020; Li et al., 2020; Wang et al., 2020c). There are several transport pathways for OPFRs in the environment, including atmospheric deposition (Mihajlovi and Fries, 2012), long-range atmospheric transport (Cheng et al., 2013), reclaimed water irrigation, and wastewater sludge application (Wei et al., 2015; Fu et al., 2017). Moreover, results from toxicity testing and risk assessments suggest that health concerns must be raised at current exposure levels for most OPFRs (Burgess et al., 2013; Blum et al., 2019).

Soil is the major reservoir and interchange for hydrophobic organic compounds (Yadav et al., 2018a). Irrigation with sewage and the application of biosolids to agricultural lands can introduce impressive OPFRs to soil ecosystems (Miller et al., 2016). Sorption and transformation are key processes for organic pollutants in the soil environment, crucial to plant's bioavailability. As organic compounds, OPFRs can readily adsorb into the soil particles due to their high hydrophobicity (log $K_{\text {ow, }}$ Table 1) (Pang et al., 2013). The amounts of dissolved OPFRs depend on the portion of OPFRs in the soil solution compared with those in soil particles. The factors affecting the bioavailability of OPFRs to plants are still unclear.
Several studies showed a positive correlation between the concentration of OPFRs in soil and the soil organic matter (SOM) content. Whereas other studies believed that the concentration of OPFRs had no significant relationship with SOM (He et al., 2017; Yadav et al., 2018a, 2018b; Zhong et al., 2018). Besides, OPFRs adsorbed into the soils may also be related to soil minerals or environmental parameters (Hao et al., 2020). Hence, it is necessary to clarify the sorption and transformation behaviors of OPFRs in the soil to provide insight into the environment's fate of OPFRS in the soil-plant system.

Besides, the safe production of plants is an essential issue to human health. Plants can accumulate OPFRs from contaminated soil and transfer them to consumers at higher trophic levels via the food chain, which poses potential ecological and human health risks. The uptake of OPFRs by plant roots and translocation to aerial tissues may be affected by the properties of OPFRs, the physiological characteristics of plants, and environmental factors. For example, though tris (1-chloro-2-propyl) phosphate (TCIPP) had similar properties to triethyl-chloro-phosphate (TCEP) to some extent, there were compound-specific uptake and accumulation patterns in plants, verified by their concentration data (Eggen et al., 2013). Meanwhile, TCEP displayed different bioconcentration factors for leaf in the meadow fescue and carrot (3.9 and 42.3, respectively) (Eggen et al., 2013). Thus, it is essential to comprehensively analyze the accumulation of OPFRs by various plant species and a discussion of affecting factors. Here, we reviewed the environmental fate and effects of OPFRs in the soil-plant system through the following aspects: (i) processes affecting the availability of OPFRs to plant roots; (ii) effects of plant physiology and chemical properties on the root uptake and translocation of OPFRs; and (iii) ecological effects of OPFRs in the soil-plant system. We also highlighted several research gaps and provided suggestions for future research, especially in situ research on the environmental fate of OPFRs.

Table 1 Physicochemical properties of selected OPFRs.

\begin{tabular}{|c|c|c|c|c|c|c|}
\hline Type & OPFRs & Solubility $\left(\mathrm{mg} \mathrm{L}^{-1}\right)$ & Vapor point $\left({ }^{\circ} \mathrm{C}\right)$ & $\log K_{\text {ow }}$ & $\mathrm{BCF}$ & $\mathrm{H}\left(25^{\circ} \mathrm{C}\right)$ \\
\hline \multirow[t]{3}{*}{ Alkyl-phosphates } & TBPs & 280 & 289 & 4 & 1030 & $1.5 \times 10^{-7}$ \\
\hline & TBOEP & 1200 & 216 & 3.65 & 1080 & $3.3 \times 10^{-11}$ \\
\hline & TEP & $5 \times 10^{5}$ & 215 & 0.8 & 3.88 & $3.5 \times 10^{-6}$ \\
\hline \multirow[t]{3}{*}{ Aryl-phosphates } & TPhP & 1.9 & 370 & 4.59 & 113 & $3.3 \times 10^{-6}$ \\
\hline & TCPs & 0.36 & 235 & 5.11 & 8560 & $7.2 \times 10^{-7}$ \\
\hline & DCPs & 0.24 & 235 & 3.6 & 391 & $1.1 \times 10^{-4}$ \\
\hline \multirow[t]{3}{*}{ Chloro alkyl-phosphates } & TCPP & 1600 & 342 & 2.59 & 8.51 & $1.9 \times 10^{-6}$ \\
\hline & TCEP & 7000 & 351 & 1.44 & 1.37 & $3.3 \times 10^{-6}$ \\
\hline & TDCPP & 1.5 & 457 & 3.8 & 13.5 & $2.6 \times 10^{-9}$ \\
\hline
\end{tabular}

Notes: $\log K_{\mathrm{ow}}$ : n-octanol/water partition coefficient; BCF: bioaccumulation factor; $\mathrm{H}$ : Henry's law constant $\left(\right.$ atm $\left.\mathrm{m}^{-3} \mathrm{~mol}^{-1}\right)$ at $25^{\circ} \mathrm{C}$; Data are compiled from previous studies (van der Veen and de Boer, 2012; Wei et al., 2015; Cristale et al., 2017). 


\section{Processes impacting availability of OPFRs to plant roots}

The environmental fate of OPFRs in the soil-plant system mainly includes sorption, transformation, root uptake, and translocation (Fig. 1). Only dissolved OPFRs in soil solution were available for root uptake by plants. Whereas, the availability of OPFRs was affected by various processes, such as sorption and transformation.

\subsection{Sorption}

Generally, organic compounds combined with the surface of the solid substance are often referred to as adsorption, while those inside the sorbent are known as partition. Both above two processes are called sorption. The sorption capacity of the organic compound to soil particles determines their contents of dissolved fractions in the soil solution, and only the latter is considered available to plants. Since most OPFRs belong to nonionic organic compounds, their dominant sorption process in the soil is considered to be partitioning into the SOM. Besides, a study demonstrated that hydrophobic interaction and $\pi-\pi$ interaction were also involved in the sorption of OPFRs in soil (Cristale et al., 2017).

Furthermore, hydrolysis intermediates of triphenyl phosphate (TPhP) were prone to protonation and form hydrogen bonds with SOM, leading to higher sorption (Wang et al., 2020a). OPFRs are neutral molecules in the soil environment, while their hydrolysis metabolites with $p K a<3$ are anions (Stevens et al., 2006). Thus, the anionic metabolites may interact with SOM by electrostatic repulsion, leading to lower sorption. Consequently, partition inside the soil and hydro- phobic interaction on the surface of soil particles were the main sorption processes for most OPFRs. In contrast, $\pi-\pi$ interaction, electrostatic interaction, and hydrogen bonding were also involved in the sorption of specific OPFRs and their hydrolysis intermediates.

Both chemical properties and soil characteristics can affect the sorption behaviors of OPFRs in soil. Hydrophobicity $\left(\log K_{\text {ow }}\right)$ is a key parameter controlling the sorption of nonionic organic compounds to SOM. The OPFRs with higher $\log K_{\text {ow }}$ values showed stronger sorption capacities to humic acid than those with lower log $K_{\text {ow }}$ (Pang et al., 2013). Besides, the lower temperature was beneficial to the sorption process of three OPFRs (tri-n-butyl phosphate (TnBP), tris(2-butoxyethyl) phosphate (TBOEP), and TCEP) in soil (Zheng et al., 2016). Cation exchange capacity or $\mathrm{pH}$ of soil may also affect organic contaminants' sorption, particularly for charged OPFRs (Hyland et al., 2015a).

Moreover, a study demonstrated that the sorption capacities of TPhP and its hydrolysis intermediates were positively correlated with sediment organic matter, zeta potential, and $\mathrm{C} / \mathrm{H}$ ratio of sediments (Wang et al., 2020a). The above results highlighted the effects of soil constituents on the sorption of OPFRs. Hence, clarification of sorption behaviors of OPFRs in the complex soil environment would help provide insight into the mechanical study on the root uptake of dissolved OPFRs by plants.

\subsection{Transformations of OPFRs in soil}

The contents of OPFRs in soil may be altered by abiotic and biotic transformation, thereby affecting plants' available OPFR contents.

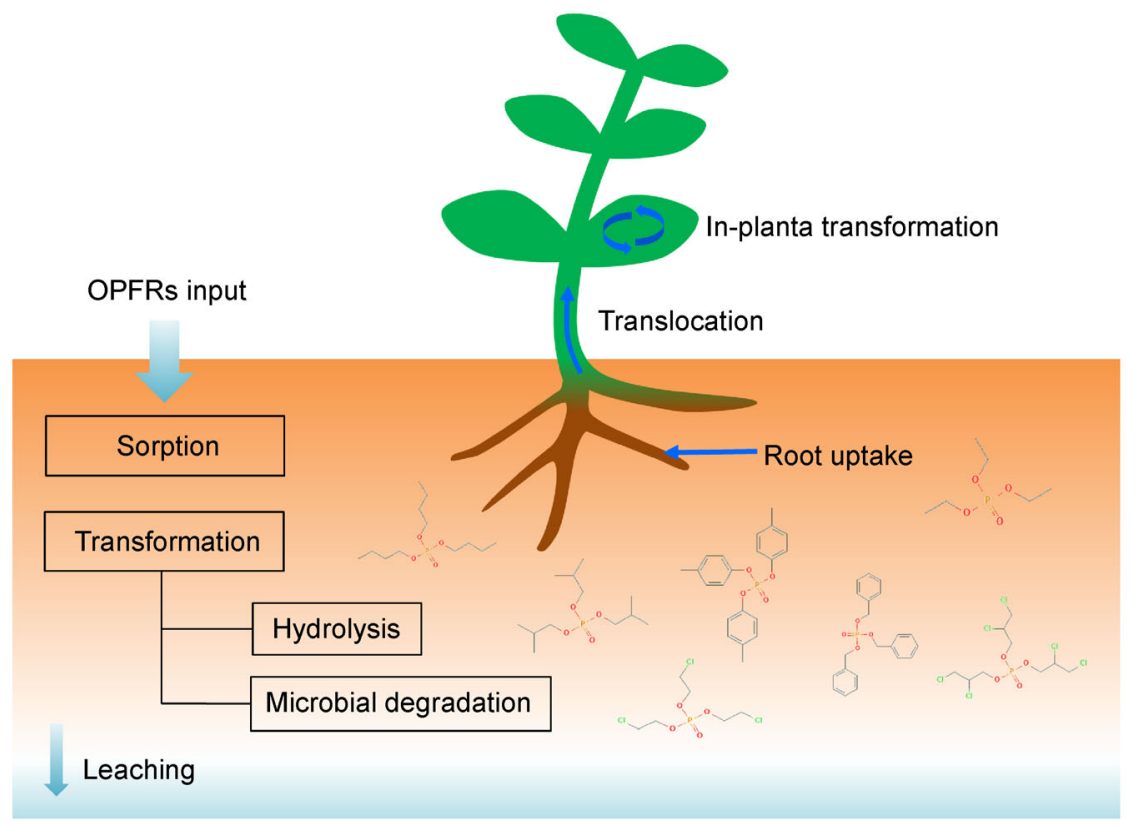

Fig. 1 Schematic showing the environmental fate of organophosphate flame retardants (OPFRs) in the soil-plant system. 


\subsubsection{Abiotic transformations}

Abiotic transformations of organic pollutants in soil include oxidation/reduction, hydrolysis, and photolysis. Although the photolysis of OPFRs in the atmosphere and solution was possible, that in the soil it is considered unrealistic due to the low vaporability of most OPFRs and light attenuation (photic soil zone < $0.5 \mathrm{~mm}$ from top) (Liu et al., 2014; Su et al., 2016). Besides, the hydrolysis of OPFRs was exceedingly slow (halflife period over two years) in the neutral homogenous solution. Still, rapid hydrolysis (half-life period less than ten days) was observed in the existence of metal (hydr)oxide minerals (Fang et al., 2018). That is, hydrolysis can be widespread in Fe-rich soil. However, Si and Al oxide minerals failed to exert a catalytic effect, indicating that OPFRs will persist in soil and aquifer systems dominated by these phases (Fang et al., 2018). Oxidation of OPFRs may also occur in soil, demonstrating that radical $\mathrm{OH}$ produced by pyrite activated persulfate could be an effective oxidant for the TCEP removal process (Saint-Hilaire et al., 2011; Lian et al., 2019).

\subsubsection{Biotic transformations}

Biotic transformation of organic pollutants may include phytoand soil animal-transformation and microbial degradation. Phytotransformation of OPFRs could occur in plants both in vivo and in vitro, which was simultaneous with root uptake and discussed in detail in Section 5.

Terrestrial animals are exposed to organic pollutants via different routes: (i) direct contact with the surrounding media dermally; (ii) the ingestion of soil particles or organic matter; and (iii) the respiratory tract when organic compounds are volatile or semi-volatile (González-Alcaraz et al., 2020). An earthworm is a typical soil animal that can intake TPhP through dermal and ingestion routes. Enzymes in earthworms for TPhP metabolism may include cytochrome P450 and phosphotriesterase, relatively by oxidative hydroxylation and hydrolysis (phase I metabolism). In contrast, phosphate conjugation dominates phase II metabolism of tributyl phosphates (TBPs) in earthworms additionally (Wang et al., 2018a, 2018b). The metabolism of OPFRs in earthworm corresponds with HepG cell in vitro metabolic experiment, in which two modules (glutathione biosynthesis and histidine degradation) and three metabolic pathways (alanine, aspartate, and glutamine metabolism; glycine, serine, and threonine metabolism; and glutamine and glutamate metabolism) were dominant (Jokanović, 2001; Gu et al., 2019). OPFRs transformed by soil animal may be detoxified to some extent, which prompts soil back to its initial condition (GonzálezAlcaraz et al., 2020).

Microbial degradation plays an essential role in OPFRs removal (Kawagoshi et al., 2002; Hurtado et al., 2017). There were several studies on the microbial degradation of OPFRs (Table 2). For example, a study on the degradation of tricresylphosphate (TCP) in agriculture soil demonstrated that non-sterilized treatment degraded approximately $80 \%$ TCP compared with approximately $45 \%$ abiotic removal in sterilized treatment after $40 \mathrm{~d}$ of incubation, suggesting that microbial activities played a vital role in the degradation of TCPs (Hurtado et al., 2017). Another study on microbial degradation consortium, GYY (dominant genera were Pseudarthrobacter and Sphingopyxis), could metabolize 92.2\% TPhP within $4 \mathrm{~h}$ (Yang et al., 2020b). Moreover, the degradation rates by Brevibacillus brevis of tri-m-cresyl phosphate, tri-p-creysl phosphate, and tri-o-cresyl phosphate were $82.9 \%, 93.9 \%$, and $53.9 \%$, respectively, within $5 \mathrm{~d}$. Chlorinated OPFRs like TCEP are the most difficult type to undergo biodegradation (Wang et al., 2019b). In addition to the molecular structure and other chemical properties of OPFRs, the real environment's external parameters should also be considered to improve the degradation rate under in situ conditions (Yang et al., 2020a).

\section{Root uptake of OPFRs}

Root uptake is always the prominent pathway for OPFRs to enter the plant due to their high vapor points (Table 1). Several factors are affecting the root uptake of OPFRs in the soil-plant system, including root physiology, transport processes, and chemical properties.

\subsection{Root physiology and transport processes}

There are three main pathways for solutes to enter the root vasculature: transmembrane (between cells through cell walls and membranes), symplastic (between cells through interconnecting plasmodesmata), and apoplastic (along cell walls through the intercellular space) routes (Miller et al., 2016). OPFRs concentrations in plant roots (carrot, mung bean, lettuce, and wheat) could increase rapidly and reach equilibrium within $12-48 \mathrm{~h}$ during a hydroponic experiment (Liu et al., 2019b). Linear concentration-dependent accumulations of OPFRs suggested passive uptake in strawberry, lettuce, carrot, and wheat (Trapp and Eggen, 2013; Hyland et al., 2015a). Most anthropogenic organic chemicals by plant roots were passive, diffusive, except for some hormone-like chemicals involved in active uptake (Collins et al., 2006). Moreover, study on the root's uptake of OPFRs exposed to metabolic inhibitors confirmed that the OPFR uptake process did not require energy (Gong et al., 2020). In the same study, the root uptake of alkyl-OPFRs, chlorinated OPFRs, and aromatic OPFRs by wheat were independent, partly mediated by, and mostly mediated by aquaporin channels, respectively. The anion channels were implicated in uptake of alkyl-OPFRs and aromatic OPFRs rather than chlorinated OPFRs (Gong et al., 2020). Therefore, the transport processes of OPFRs were compound-specific.

Root physiology also affects the root uptake of OPFRs, including the protein and lipid contents in plants (Liu et al., $2019 b$ ). Uptake into carrot roots is higher than barley roots in greenhouse experiments, which might be attributed to the higher lipid content of carrots (Trapp and Eggen, 2013). 
Table 2 Microbial degradation of OPFRs.

\begin{tabular}{llll}
\hline Microorganisms & Substrates \& degradation efficiency & Main results & Reference \\
\hline A mixed bacterial population isolated & TCrP, TPhP, trixylenyl phosphate & No evidence of extracellular & Pickard et al., 1975 \\
from mud sample of shell lake & $($ TXP) & enzymes capable of triaryl \\
phosphate degradation
\end{tabular}

Naturally occurring mixed-microbial populations present in activated sludge and river water

Leachate from a sea-based solid waste disposal site, Osaka North Port, Japan

Roseobacter YS-57, isolated from the leachate of a sea-based waste disposal site, Osaka North Port, Japan

Mixed bacterial cultures $67 \mathrm{E}$ and $45 \mathrm{D}$ enriched from soil and sediment, Niigata, Japan

Sphingomonas sp. TCM1 and Sphingobium sp. TDK1, isolated from soil and sediment samples, Niigata, Japan

Activated sludge from either the Amsterdam West or the Amstelveen sewage treatment plants, US

Brevibacillus brevis, isolated from an e-waste dismantling area in Guiyu, Guangdong Province, China

Sphingomonas sp., isolated from the tailing sand of Hechi City, Guangxi Province, China

Microcosms inoculated with contaminated river sediments receiving wastewater from a halogenated OPFRs production plant in Hebei Province, China

A comprehensive 3D-QSAR model to evaluate 17 OPFRs biodegradation effects
TCP,TBP, and TPP showed complete primary degradation in 7 days

Aryl-phosphates (TCrP, TPHP), alkyl-phosphates (TnBP), cholo-alkyl phosphates (TCEP, TDCPP)

TCrP, TPhP (0.5 mg L ${ }^{-1}$, over $99 \%$ within 3 days)

Completely degraded $20 \mu \mathrm{mol} \mathrm{L}^{-1}$ TCEP and TDCPP within $6 \mathrm{~h}$

TCEP and TDCPP $(20 \mu \mathrm{mol} \mathrm{L}-1$, $100 \%$, as the sole phosphorus source within $6 \mathrm{~h}$ )

Aromatic organophosphorus flame retardants TPhP $\left(20 \mathrm{mg} \mathrm{L}^{-1}\right.$, $99.0 \% \pm 24.3 \%$ within $28 \mathrm{~h}$ )

TPhP (the highest degradation efficiency of $3 \mu \mathrm{mol} \mathrm{L}{ }^{-1}$ TPhP by $B$. brevis reached $92.1 \%$ at $\mathrm{pH} 7$ and $30^{\circ} \mathrm{C}$ )

TnBP could be utilized as the sole carbon source (375 $\mu \mathrm{mol} \mathrm{L}^{-1}$, over $90 \%, 63 \mathrm{~h}$ )

Completely degraded $20 \mathrm{mg} \mathrm{L}^{-1}$ TCEP within $96 \mathrm{~h}$; TCEP degradation fitted the pseudo-zero-order kinetics with a reaction rate of $0.068 \mathrm{mg} \mathrm{L}^{-1} \mathrm{~h}^{-1}$

Biodegradation efficiency of the TCPP-OH increased by $75.52 \%$ compared to TCPP under simulated environment
Most likely involving a

Victor et al., 1979 stepwise enzymatic hydrolysis to orthophosphate and the phenolic or alcohol moieties

No observed decrease of OPEs under anaerobic

Kawagoshi et al., under anaerobic 2002

condition except of aryl-phosphates

APE-degrading enzyme was released into the

Kawagoshi et al., 2004

extracellular space in the logarithmic growth phase

The cleavage of the phosphor ester bond and Takahashi et al., 2008 dehalogenation related with Sphingomonas- and

Acidovorax-related bacteria

Hydrolyzing their phosphotriester Takahashi et al., bonds, generating 2010

2-CE, and 1,3-DCP as metabolites from TCEP and TDCPP

Conversion of TPhP to Jurgens et al., 2014

DPP by hydrolysis of a

phosphate ester linkage; DPP was degraded further in non-sterilized sludge

Bioaugmentation with Wei et al., 2019 degrading bacteria stimulated the degradation of TPhP in river water

The first irreversible step of TBP Liu et al., 2019a transferred to DnBP would lead to $\mathrm{P}-\mathrm{O}$ bond cleavage

Mainly degraded via Zhou et al., 2020 phosphoester bond hydrolysis; hydrolytic dechlorination and oxidation pathways might involve

On simulating the biodegradation Yang et al., 2020a path and photodegradation path, it is found that the energy barrier of TCPP $-\mathrm{OH}$ for phosphate bond cleavage is reduced by $15.73 \%$ and $52.52 \%$ compared to TCPP after modification 
Furthermore, there are large number of functional proteins in the plant apoplast. For example, TaLTP1.1 is one of the most important nonspecific lipid transfer proteins (nsLTPs) in wheat. OPFRs with higher hydrophobicity were more liable to bind with TaLTP1.1, leading to their excellent uptake rate in wheat roots (Liu et al., 2019b).

\subsection{Relationship between root uptake and chemical properties}

Root-soil concentration factors (RCFs, $\mathrm{C}_{\text {root }} / \mathrm{C}_{\text {soil }}$ ) and rootwater concentration factors $\left(\mathrm{RCF}_{\mathrm{w}}, \mathrm{C}_{\text {root }} / \mathrm{C}_{\text {water }}\right)$ can indicate plant roots' bioaccumulation ability of contaminants from soil and water, respectively. A hydroponic experiment showed that $\operatorname{logRCF} \mathrm{F}_{\mathrm{w}}$ values of OPFRs positively correlated with their $\log K_{\text {ow }}$, indicating that higher hydrophobic OPFRs could be accumulated easier by plants (Wan et al., 2017). Our analysis of published data also showed that the $\operatorname{logRCF}_{\mathrm{w}}$ was significantly correlated with $\log K_{\text {ow }}$ of OPFRs $(p<0.001$, Fig. 2A, based on R package "basicTrendline"). However, the $\log R C F s$ did not show a significant correlation with $\log K_{\text {ow }}$ $(p=0.235$, Fig. 2A). This is also reasonable because soil particles would compete with plant roots for dissolved OPFRs in soil solution. Furthermore, the variability of SOM will also greatly impact the root uptake of OPFRs, resulting in no significant correlations observed under the soil culture condition. Hence, it should focus more on the root uptake of OPFRs in the soil environment by considering the effects of complicated SOM components and other soil constituents.

\section{Accumulation of OPFRs in aerial tissue}

The accumulation of organic compounds in the aboveground tissues of plants may result from a combination of translocation from roots and foliar uptake from the air, even though the latter one is always negligible. Organic compounds may move through roots to shoots via transpiration stream or be blocked by the Casparian strip in roots. The transpiration stream concentration factor (TSCF, $\mathrm{C}_{\text {transpiration stream }} / \mathrm{C}_{\text {solution}}$ ) is always used to describe the acropetal translocation ability of organic compounds. Our published data analysis showed that the TSCF values were negatively correlated with their $\log K_{\text {ow }}$ values ( $p=0.001$, Fig. $2 \mathrm{~B}$ ). In contrast, it was found that the TSCF was at a maximum for nonionic organic chemicals (e.g., polybrominated biphenyls) with $\log K_{\text {ow }}$ around 2 to 3 (Collins et al., 2006). The conflict between these conclusions might be attributed to limited experimental data on OPFRs. The translocation factor ( $\mathrm{TF}, \mathrm{C}_{\text {aboveground }} / \mathrm{C}_{\text {root }}$ ) is also used to reflect the translocation ability of compounds from roots to aboveground tissues. The TF values were also significantly negatively correlated with $\log K_{\text {ow }}$ values of OPFRs $(p=0.015$, Fig. $2 \mathrm{C}$ ). Thus, higher hydrophobic OPFRs were steadily distributed in roots and were difficult to be translocated to aboveground tissues. Neutral compounds with $\log K_{\text {ow }}$ of 0.5 3.0 are prone to be translocated (Gong et al., 2020; Liu et al., 2020a). For example, TCEP concentrations in shoots were significantly higher than root concentrations during exposure, indicating efficient translocation from roots to shoots in wheat seedlings (Gong et al., 2020). Nevertheless, the above correlations between TSCF (or TF) and log $K_{\text {ow }}$ were analyzed using only data from hydroponic experiments. More experiments under soil culture conditions were suggested and helpful for better understanding the uptake mechanisms of OPFRs by plants. Also, the uptake and accumulation by crops were evidenced by field investigation, which may provide the potential for OPFRs to transfer into the food chain and pose threats to human health (Wan et al., 2016).

\section{In planta transformations}

In planta transformation of xenobiotic pollutants is critical for detoxification mechanisms. The transformation of OPFRs in planta and rhizosphere is similar to microbial transformation

\section{(A)}

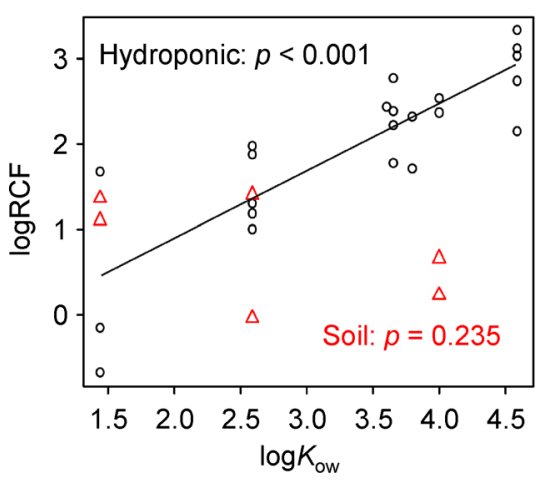

(B)

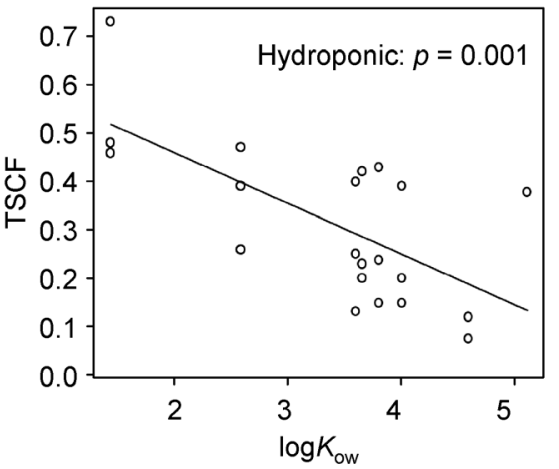

(C)

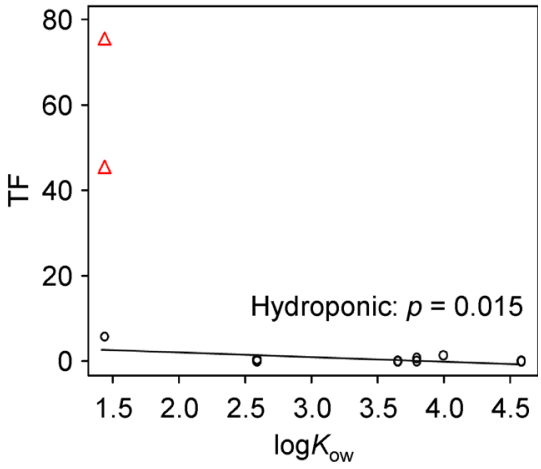

Fig. 2 Relationships between $\log R C F(A), T S C F(B)$, and TF (C) values and log $K_{\text {ow }}$ values of OPFRs. RCF, root concentration factor; TSCF, transpiration stream concentration factor; and TF, translocation factor. Correlation analysis and visualization were performed using R package "basicTrendline" (Mei et al., 2018). Black dots represent data under hydroponic conditions, and red triangles represent data under soil culture conditions. Data were cited from (Collins and Finnegan, 2010; Eggen et al., 2013; Hyland et al., 2015b; Wan et al., 2016; Wan et al., 2017; Liu et al., 2019b; Wang et al., 2019; Gong et al., 2020). 
reactions in soil and sediments, composed of hydrolysis, dealkylation, oxidative dichlorination, hydroxylation, methoxylation, and glutathione-, and glucuronide-conjugation (Wan et al., 2017; Dobslaw et al., 2021; Gong et al., 2020). The metabolism of OPFRs in wheat occurred very rapidly because the metabolites were detected in wheat tissues after OPFRs exposure for $12 \mathrm{~h}$ (Wan et al., 2017). The hydrolysis of TCEP might occur mainly in roots. Then, hydrolysis metabolites of TCEP (BCEP) were transported to the shoots because concentrations of TCEP in both root and shoot decreased, while BCEP concentration in shoots increased when exposed to TCEP (Gong et al., 2020). Chlorinated OPFRs could only be transformed into diesters, while non-chlorinated OPFRs could further transform diesters into monoesters (Wan et al., 2017). Another biological pathway analysis indicated that three major metabolic pathways: alanine, aspartate, and glutamate metabolism; glyoxylate, and dicarboxylate metabolism; and tricarboxylic acid cycle (TCA cycle) were disturbed when wheat was exposed to the chlorinated OPFRs (Liu et al., 2020a).

The root exudates released during plant cultivation would result in the metabolism of OPFRs in the rhizosphere to some extent (Wan et al., 2017). The metabolite of TnBP (DnBP) concentrations was in the order: hydroponic solution>root>shoot, which implied that the uptake from hydroponic solution was another source for some metabolites in plants (Gong et al., 2020). Since OPFRs hydrolysis metabolites with relatively high proportions in electrical repulsion of the cytomembrane, it was supposed that cell walls could be a location for in vivo hydrolysis of OPFRs by wheat (Gong et al., 2020).

\section{Ecological effects of OPFRs in the soil-plant system}

The ecological effects of OPFRs were discussed in three aspects: soil microorganisms, plants, and animals (Fig. 3). Microorganisms account for a huge amount of the soil. The balance between endogenous and exogenous reactive oxygen species (ROS) in microorganisms could be disturbed when exposed to exotic pollutants, including OPFRs. Therefore, excessive production of ROS incurred mitochondrial dysfunction, protein peroxidation, and DNA damage. Besides, the OPFR isomers could pose oxidative damage to a different extent. For TCP isomers, the largest ROS increment was observed in tri-o-cresylphosphate (ToCP) exposure, which in turn imposed influences on the metabolic process of ToCP. The degradation efficiency of ToCP was remarkably lower than those of the other isomers (Liu et al., 2019c). The microbial richness and diversity of rhizosphere soil were significantly reduced after short-time exposure to OPFRs. These rhizospheric microbes were mainly grouped by plant effects due to specific root exudates and rhizodeposition that nourished rhizosphere microorganisms. Still, a more obvious deviation of microorganisms in the soil was discovered after exposure to OPFRs, regardless of plant species. Furthermore, analysis of the shifts in rhizosphere nitrogen transformation-associated genera after OPFRs treatment suggested OPFRs loading reduced ammonia-oxidizing bacterial levels and inhibited the $\mathrm{NH}_{4}{ }^{+}-\mathrm{N}$ removal. In contrast, the enriched Pseudomonas in the rhizosphere did not ameliorate total nitrogen removal (Liu et al., 2020b).

The metabolic responses of plants under OPFRs stress are important to understand their potential risks to terrestrial organisms. Wheat exposed to three chlorinated OPFRs for $7 \mathrm{~d}$ had decreased chlorophyll content, while the levels of carotenoid and activities of antioxidases, malonaldehyde, and ROS increased in both roots and shoots, indicating that chlorinated OPFRs caused significant oxidative stresses and affected photosynthesis in wheat (Liu et al., 2020a). The aspartate family plays a vital role in the transport and storage of nitrogen in organisms. The upregulation of aspartic acid and asparagine in wheat cultivation experiment exposure by chlorinated OPFRs implied that nitrogen assimilation in wheat

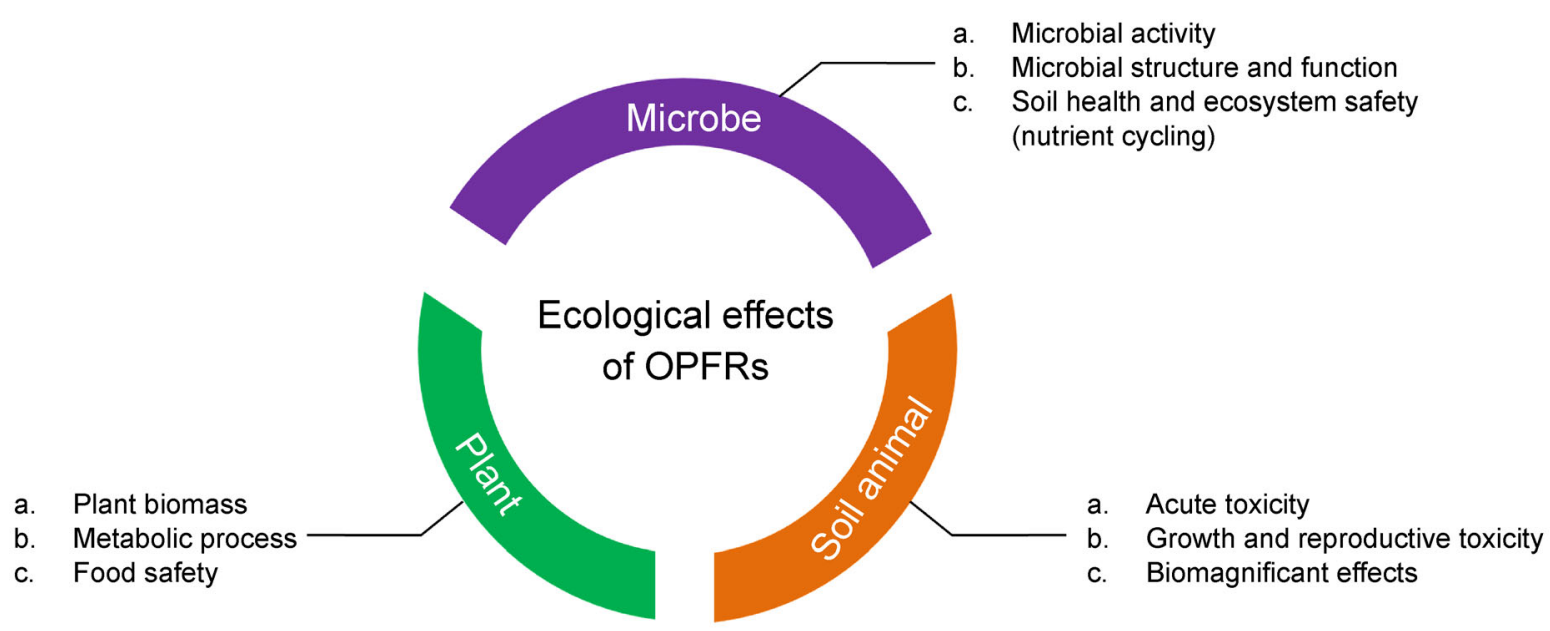

Fig. 3 Schematic showing the potential ecological effects of OPFRs in the soil-plant system. 
was disturbed (Liu et al., 2020a). Except for higher plants, numerous microalgae play a role as primary producers in a humid soil environment. Previous studies showed that OPFRs may have similar toxic potential to microalgae, exposure to tris (1,3-dichloroisopropyl) phosphate (TDCIPP), and TPhP resulted in a dose-response decrease of microalgae's biomass and plasma membrane damage (Wang et al., 2019b; Wang et al., 2020a). Integrating the computer simulation technology and biological approach investigated the biological effects of OPFRs on microalgae, revealed a positive correlation with cell inhibition alongside the incremental chain length of alkyl-OPFRs (Chu et al., 2021).

Earthworms play essential roles in soil ecosystems. The toxic effects of OPFRs in exposed earthworms may be useful for the risk assessment of OPFRs. Some studies have proved that OPFRs could induce oxidative stress in earthworms (Wang et al., 2018a, 2018b; Mennillo et al., 2019). The effect on bodyweight of TCEP and TCP (10 mg kg-1, both) was low in exposed earthworms (Yang et al., 2018). A chronic exposure experiment showed that TDCIPP treatments (over $500 \mathrm{ng} \mathrm{g}^{-1}$ ) lowered body mass and juveniles born rate of earthworms, and seminal vesicle injury, skin lesions, and muscle atrophy were observed as well (Zhu et al., 2019). The observed changes in AchE activity and the analysis of genes showed that TCEP and TCP caused intestinal damage to earthworms (Yang et al., 2018). Prolonged exposure to TDCIPP (1 $\mathrm{mg} \mathrm{kg}^{-1}$ ) accelerated the aging process of Caenorhabditis elegans, including decreased locomotion behaviors, shortened lifespan, lipofuscin, and lipid peroxidation accumulation (Wang C. et al., 2019a). We deduced that the toxic dosage of OPFRs was compound-specific, but their toxic mechanisms were similar. Also, insects and amphibians have been studied under OPFRs exposure conditions and contributed to the transfer of OPFRs in the terrestrial food chain (Poma et al., 2019; Liu et al., 2020c). Risk assessments conducted by the European Commission in 2009 claimed that since there was no indication that TCEP/TCPP might show a bioaccumulation potential, risk characterization for exposure via the food chain was unnecessary (Trapp and Eggen, 2013). However, based on the experimental bioconcentration factors for TCEP and TCPP, the high variation between species and a persistency potential, there is reason to investigate the transfer and possible bioaccumulation of OPFRs in terrestrial food webs more deeply.

\section{Conclusions and future perspectives}

Historically, except for insecticides, organophosphate esters are widely applied as flame retardants and plasticizers, particularly after the implementation of regulations that phased out PBDEs. Until now, OPFRs are often detected at higher levels in environmental matrices than PBDEs, and results from toxicity testing and risk assessments suggest that health concerns must be raised at current exposure levels for most OPFRs. Clarification of the sorption behaviors of OPFRs is crucial to predict their bioavailability and to develop emerging pollutants control methods. Partition inside the soil and hydrophobic interaction on the surface of soil particles were the main sorption processes for most OPFRs. In contrast, $\pi-\pi$ interaction, electrostatic interaction, and hydrogen bonding were also involved in the sorption of specific OPFRs and their hydrolysis intermediates. Besides, hydrolysis and microbial degradation may be important pathways for OPFRs to be transformed in soil. The distribution of OPFRs between soil particles and plant roots determines the root uptake behaviors. Hence, the rhizosphere is the key area that needs to be focused on in the soil-plant system. DNA-stable isotope probing is a powerful technique for identifying active microorganisms capable of degrading contaminants; thus, it is suggested for a more complete understanding of the rhizospheric microbial communities. Correlation analyses on published data indicated that hydrophobicity $\left(\log K_{\mathrm{ow}}\right)$ was the dominant factor controlling the root uptake of OPFRs (logRCFw) by plants under hydroponic conditions. SOM and other soil constituents greatly affect the root uptake of OPFRs by plants under soil culture conditions, resulting in no significant correlation between logRCFs and $\log K_{\text {ow. }}$. Translocation of OPFRs from roots to aboveground tissues decreased with increased OPFRs' hydrophobicity, which was confirmed by the correlation analyses between TSCF (or TF) and $\log K_{\text {ow }}$ using data from the hydroponic experiment. More experiments under soil culture conditions were suggested and helpful for better understanding the uptake mechanisms of OPFRs by plants. Also, the transformation of OPFRs in plant tissues was also possible, in which hydrolysis plays a significant role. Potential ecological risks of OPFRs in terrestrial environments should also be considered.

Future study is suggested to focus on the following aspects: (i) Increasing field experiment to simulate key factors controlling the bioavailability of OPFRs, and developing computative methods to predict the bioavailability of OPFRs; (ii) paying attention to the effects of root exudates on root uptake of OPFRs; (iii) concerning the response and mechanisms of various plant rhizospheric microorganisms to OPFRs; (iv) establishing and improving the risk threshold of OPFRs to the ecosystem and human; and ( $v$ ) developing green and sustainable technologies for in situ remediation of OPFRscontaminated soil, including the combined technologies.

\section{Conflict of interest}

The authors declare no conflict of interest.

\section{Acknowledgments}

Financial support was provided by the Local Innovative and Research Teams Project of Guangdong Pearl River Talents Program (2017BT01Z134), the National Natural Science Foundation of China (32061133003 and 41603086), the Guangdong 
Foundation for Program of Science and Technology Research (2019B121205006), and Ten Thousand Talent Program of the Organization Department of the Central Committee of the CPC.

\section{Author contributions}

Qing Zhang: Investigation, Writing- Origianl Draft, Visualization. Weiping Mei: Investigation, Writing- Origianl Draft, Visualization. Longfei Jiang: Investigation, Writing- Review \& Editing. Qian Zheng: Writing- Review \& Editing. Chunling Luo: Conceptualization, Supervision, Writing- Review \& Editing. Gan Zhang: Conceptualization, Supervision.

\section{References}

Blum, A., Behl, M., Birnbaum, L., Diamond, M.L., Phillips, A., Singla, V., Sipes, N.S., Stapleton, H.M., Venier, M., 2019. Organophosphate ester flame retardants: Are they a regrettable substitution for polybrominated diphenyl ethers? Environmental Science \& Technology Letters 6, 638-649.

Burgess, R.M., Ho, K.T., Brack, W., Lamoree, M., 2013. Effectsdirected analysis (EDA) and toxicity identification evaluation (TIE): Complementary but different approaches for diagnosing causes of environmental toxicity. Environmental Toxicology and Chemistry 32, 1935-1945.

Chen, Y., Song, Y., Chen, Y.J., Zhang, Y., Li, R., Wang, Y., Qi, Z., Chen, Z.F., Cai, Z., 2020. Contamination profiles and potential health risks of organophosphate flame retardants in $\mathrm{PM}_{2.5}$ from Guangzhou and Taiyuan, China. Environment International 134, 105343.

Cheng, W., Sun, L., Huang, W., Ruan, T., Xie, Z., Zhang, P., Ding, R., $\mathrm{Li}, \mathrm{M} ., 2$ 2013. Detection and distribution of tris (2-chloroethyl) phosphate on the East Antarctic ice sheet. Chemosphere 92, 1017-1021.

Chu, Y., Zhang, C., Ho, S.H., 2021. Computational simulation associated with biological effects of alkyl organophosphate flame retardants with different carbon chain lengths on Chlorella pyrenoidosa. Chemosphere 263, 127997.

Collins, C., Fryer, M., Grosso, A., 2006. Plant uptake of non ionic organic chemicals. Environmental Science \& Technology 40, 4552.

Collins, C.D., Finnegan, E., 2010. Modeling the plant uptake of organic chemicals, including the soil-air-plant pathway. Environmental Science \& Technology 44, 998-1003.

Cristale, J., Álvarez-Martín, A., Rodríguez-Cruz, S., Sánchez-Martín, M.J., Lacorte, S., 2017. Sorption and desorption of organophosphate esters with different hydrophobicity by soils. Environmental Science and Pollution Research International 24, 27870-27878.

Dobslaw, D., Woiski, C., Kiel, M., Kuch, B., Breuer, J., 2021. Plant uptake, translocation and metabolism of PBDEs in plants of food and feed industry: A review. Reviews in Environmental Science and Bio/Technology 20, 75-142.

Eggen, T., Heimstad, E.S., Stuanes, A.O., Norli, H.R., 2013. Uptake and translocation of organophosphates and other emerging contaminants in food and forage crops. Environmental Science and Pollution Research International 20, 4520-4531.

Fang, Y., Kim, E., Strathmann, T.J., 2018. Mineral- and basecatalyzed hydrolysis of organophosphate flame retardants: Potential major fate-controlling sink in soil and aquatic environments. Environmental Science \& Technology 52, 1997-2006.

Fu, L., Du, B., Wang, F., Lam, J.C.W., Zeng, L., Zeng, E.Y., 2017. Organophosphate triesters and diester degradation products in municipal sludge from wastewater treatment plants in China: Spatial patterns and ecological implications. Environmental Science \& Technology 51, 13614-13623.

Gong, X., Wang, Y., Pu, J., Zhang, J., Sun, H., Wang, L., 2020. The environment behavior of organophosphate esters (OPEs) and diesters in wheat (Triticum aestivum L.): Uptake mechanism, in vivo hydrolysis and subcellular distribution. Environment International 135, 105405.

González-Alcaraz, M.N., Malheiro, C., Cardoso, D.N., Prodana, M., Morgado, R.G., van Gestel, C.A.M., Loureiro, S., 2020. Bioaccumulation and Toxicity of Organic Chemicals in Terrestrial Invertebrates. In: Ortega-Calvo, J.J., Parsons, J.R., eds. Bioavailability of Organic Chemicals in Soil and Sediment. Springer International Publishing, Cham, pp. 149-189.

Gu, J., Su, F., Hong, P., Zhang, Q., Zhao, M., 2019. ${ }^{1} \mathrm{H}$ NMR-based metabolomic analysis of nine organophosphate flame retardants metabolic disturbance in Hep G2 cell line. Science of the Total Environment 665, 162-170.

Hao, M., Gao, P., Yang, D., Chen, X., Xiao, F., Yang, S., 2020. Highly efficient adsorption behavior and mechanism of Urea$\mathrm{Fe}_{3} \mathrm{O}_{4} @ \mathrm{LDH}$ for triphenyl phosphate. Environmental Pollution 267, 114142.

He, M.J., Yang, T., Yang, Z.H., Li, Q., Wei, S.Q., 2017. Occurrence and distribution of organophosphate esters in surface soil and street dust from Chongqing, China: Implications for human exposure. Archives of Environmental Contamination and Toxicology 73, 349361.

Hurtado, C., Montano-Chávez, Y.N., Domínguez, C., Bayona, J.M., 2017. Degradation of emerging organic contaminants in an agricultural soil: Decoupling biotic and abiotic processes. Water, Air, and Soil Pollution 228, 243.

Hyland, K.C., Blaine, A.C., Dickenson, E.R., Higgins, C.P., 2015a. Accumulation of contaminants of emerging concern in food cropspart 1: Edible strawberries and lettuce grown in reclaimed water. Environmental Toxicology and Chemistry 34, 2213-2221.

Hyland, K.C., Blaine, A.C., Higgins, C.P., 2015b. Accumulation of contaminants of emerging concern in food crops-part 2: Plant distribution. Environmental Toxicology and Chemistry 34, 22222230.

Jokanović, M., 2001. Biotransformation of organophosphorus compounds. Toxicology and Applied Pharmacology 166, 139-160.

Jurgens, S.S., Helmus, R., Waaijers, S.L., Uittenbogaard, D., Dunnebier, D., Vleugel, M., Kraak, M.H., de Voogt, P., Parsons, J.R., 2014. Mineralisation and primary biodegradation of aromatic organophosphorus flame retardants in activated sludge. Chemosphere 111, 238-242.

Kawagoshi, Y., Nakamura, S., Fukunaga, I., 2002. Degradation of organophosphoric esters in leachate from a sea-based solid waste disposal site. Chemosphere 48, 219-225. 
Kawagoshi, Y., Nakamura, S., Nishio, T., Fukunaga, I., 2004. Isolation of aryl-phosphate ester-degrading bacterium from leachate of a sea-based waste disposal site. Journal of Bioscience and Bioengineering 98, 464-469.

Li, Y., Yao, C., Zheng, Q., Yang, W., Niu, X., Zhang, Y., Lu, G., 2020. Occurrence and ecological implications of organophosphate triesters and diester degradation products in wastewater, river water, and tap water. Environmental Pollution 259, 113810.

Lian, W., Yi, X., Huang, K., Tang, T., Wang, R., Tao, X., Zheng, Z., Dang, Z., Yin, H., Lu, G., 2019. Degradation of tris(2-chloroethyl) phosphate (TCEP) in aqueous solution by using pyrite activating persulfate to produce radicals. Ecotoxicology and Environmental Safety $174,667-674$.

Liu, J., Lin, H., Dong, Y., Li, B., 2019a. Elucidating the biodegradation mechanism of tributyl phosphate (TBP) by Sphingomonas sp. isolated from TBP-contaminated mine tailings. Environmental Pollution 250, 284-291.

Liu, Q., Liu, M., Wu, S., Xiao, B., Wang, X., Sun, B., Zhu, L., 2020a. Metabolomics reveals antioxidant stress responses of wheat (Triticum aestivum L.) exposed to chlorinated organophosphate esters. Journal of Agricultural and Food Chemistry 68, 6520-6529.

Liu, Q., Wang, X., Yang, R., Yang, L., Sun, B., Zhu, L., 2019b. Uptake kinetics, accumulation, and long-distance transport of organophosphate esters in plants: Impacts of chemical and plant properties. Environmental Science \& Technology 53, 4940-4947.

Liu, T., Lu, S., Wang, R., Xu, S., Qin, P., Gao, Y., 2020b. Behavior of selected organophosphate flame retardants (OPFRs) and their influence on rhizospheric microorganisms after short-term exposure in integrated vertical-flow constructed wetlands (IVCWs). Science of the Total Environment 710, 136403.

Liu, Y., Liggio, J., Harner, T., Jantunen, L., Shoeib, M., Li, S.M., 2014. Heterogeneous $\mathrm{OH}$ initiated oxidation: a possible explanation for the persistence of organophosphate flame retardants in air. Environmental Science \& Technology 48, 1041-1048.

Liu, Y., Yin, H., Wei, K., Peng, H., Lu, G., Dang, Z., 2019c. Biodegradation of tricresyl phosphate isomers by Brevibacillus brevis: Degradation pathway and metabolic mechanism. Chemosphere 232, 195-203.

Liu, Y.E., Luo, X.J., Guan, K.L., Huang, C.C., Zhu, C.Y., Qi, X.M., Zeng, Y.H., Mai, B.X., 2020c. Legacy and emerging organophosphorus flame retardants and plasticizers in frogs: Sex difference and parental transfer. Environmental Pollution 266, 115336.

Mei, W., Yu, G., Lai, J., Rao, Q., Umezawa, Y., 2018. basicTrendline: Add Trendline and Confidence Interval of Basic Regression Models to Plot. R package version 2.0.3. http://CRAN.R-project. org $/$ package $=$ basicTrendline

Mennillo, E., Cappelli, F., Arukwe, A., 2019. Biotransformation and oxidative stress responses in rat hepatic cell-line (H4IIE) exposed to organophosphate esters (OPEs). Toxicology and Applied Pharmacology 371, 84-94

Mihajlovi, I., Fries, E., 2012. Atmospheric deposition of chlorinated organophosphate flame retardants (OFR) onto soils. Atmospheric Environment 56, 177-183.

Miller, E.L., Nason, S.L., Karthikeyan, K.G., Pedersen, J.A., 2016. Root Uptake of pharmaceuticals and personal care product ingredients. Environmental Science \& Technology 50, 525-541.
Pang, L., Liu, J., Yin, Y., Shen, M., 2013. Evaluating the sorption of organophosphate esters to different sourced humic acids and its effects on the toxicity to Daphnia magna. Environmental Toxicology and Chemistry 32, 2755-2761.

Pickard, M.A., Whelihan, J.A., Westlake, D.W., 1975. Utilization of triaryl phosphates by a mixed bacterial population. Canadian Journal of Microbiology 21, 140-145.

Poma, G., Liu, Y., Cuykx, M., Tang, B., Luo, X.J., Covaci, A., 2019. Occurrence of organophosphorus flame retardants and plasticizers in wild insects from a former e-waste recycling site in the Guangdong Province, South China. Science of the Total Environment 650, 709-712.

Saint-Hilaire, D., Ismail, K.Z., Jans, U., 2011. Reaction of tris(2chloroethyl)phosphate with reduced sulfur species. Chemosphere 83, 941-947.

Stevens, R., van Es, D.S., Bezemer, R., Kranenbarg, A., 2006. The structure-activity relationship of fire retardant phosphorus compounds in wood. Polymer Degradation \& Stability 91, 832-841.

Su, G., Letcher, R.J., Yu, H., 2016. Organophosphate flame retardants and plasticizers in aqueous solution: $\mathrm{pH}$-dependent hydrolysis, kinetics, and pathways. Environmental Science \& Technology 50, 8103-8111.

Takahashi, S., Kawashima, K., Kawasaki, M., Kamito, J., Endo, Y., Akatsu, K., Horino, S., Yamada, R.H., Kera, Y., 2008. Enrichment and characterization of chlorinated organophosphate esterdegrading mixed bacterial cultures. Journal of Bioscience and Bioengineering 106, 27-32.

Takahashi, S., Satake, I., Konuma, I., Kawashima, K., Kawasaki, M., Mori, S., Morino, J., Mori, J., Xu, H., Abe, K., Yamada, R.H., Kera, Y., 2010. Isolation and identification of persistent chlorinated organophosphorus flame retardant-degrading bacteria. Applied and Environmental Microbiology 76, 5292-5296.

Trapp, S., Eggen, T., 2013. Simulation of the plant uptake of organophosphates and other emerging pollutants for greenhouse experiments and field conditions. Environmental Science and Pollution Research International 20, 4018-4029.

van der Veen, I., de Boer, J., 2012. Phosphorus flame retardants: properties, production, environmental occurrence, toxicity and analysis. Chemosphere 88, 1119-1153.

Victor, W., 1979. Environmental fate of selected phosphate esters. Environmental Science \& Technology 13, 840-844.

Vila-Costa, M., Sebastián, M., Pizarro, M., Cerro-Gálvez, E., Lundin, D., Gasol, J.M., Dachs, J., 2019. Microbial consumption of organophosphate esters in seawater under phosphorus limited conditions. Scientific Reports 9, 233.

Wan, W., Huang, H., Lv, J., Han, R., Zhang, S., 2017. Uptake, translocation, and biotransformation of organophosphorus esters in wheat (Triticum aestivum L.). Environmental Science \& Technology 51, 13649-13658.

Wan, W., Zhang, S., Huang, H., Wu, T., 2016. Occurrence and distribution of organophosphorus esters in soils and wheat plants in a plastic waste treatment area in China. Environmental Pollution 214, 349-353.

Wang, C., An, J., Bai, Y., Li, H., Chen, H., Ou, D., Liu, Y., 2019. Tris (1,3-dichloro-2-propyl) phosphate accelerated the aging process induced by the 4-hydroxynon-2-enal response to reactive oxidative 
species in Caenorhabditis elegans. Environmental Pollution 246, 904-913.

Wang, J., Li, X., Wu, W., Fan, S., Jia, Y., Wang, J., Yan, Y., 2019. Characterization and 16S rRNA gene-based metagenomic analysis of the organophosphorous flame retardants degrading consortium YC-BJ1. Chinese Journal of Biotechnology 35, 2050-2060 (in Chinese).

Wang, L., Huang, X., Laserna, A.K.C., Li, S.F.Y., 2018a. Metabolism of tri-n-butyl phosphate in earthworm Perionyx excavatus. Environmental Pollution 234, 389-395.

Wang, L., Huang, X., Laserna, A.K.C., Li, S.F.Y., 2018b. Untargeted metabolomics reveals transformation pathways and metabolic response of the earthworm Perionyx excavatus after exposure to triphenyl phosphate. Scientific Reports 8, 16440.

Wang, P., Li, D., Fan, X., Hu, B., Wang, X., 2020a. Sorption and desorption behaviors of triphenyl phosphate (TPhP) and its degradation intermediates on aquatic sediments. Journal of Hazardous Materials 385, 121574.

Wang, Q., Zhao, H., Xu, L., Wang, Y., 2019. Uptake and translocation of organophosphate flame retardants (OPFRs) by hydroponically grown wheat (Triticum aestivum L.). Ecotoxicology and Environmental Safety 174, 683-689.

Wang, X., Zhu, Q., Yan, X., Wang, Y., Liao, C., Jiang, G., 2020b. A review of organophosphate flame retardants and plasticizers in the environment: Analysis, occurrence and risk assessment. Science of the Total Environment 731, 139071.

Wang, Y., Yao, Y., Han, X., Li, W., Zhu, H., Wang, L., Sun, H., Kannan, K., 2020c. Organophosphate di- and tri-esters in indoor and outdoor dust from China and its implications for human exposure. Science of the Total Environment 700, 134502.

Wei, G.L., Li, D.Q., Zhuo, M.N., Liao, Y.S., Xie, Z.Y., Guo, T.L., Li, J.J., Zhang, S.Y., Liang, Z.Q., 2015. Organophosphorus flame retardants and plasticizers: sources, occurrence, toxicity and human exposure. Environmental Pollution 196, 29-46.

Wei, K., Yin, H., Peng, H., Lu, G., Dang, Z., 2019. Bioremediation of triphenyl phosphate in river water microcosms: Proteome alteration of Brevibacillus brevis and cytotoxicity assessments. Science of the Total Environment 649, 563-570.

Yadav, I.C., Devi, N.L., Li, J., Zhang, G., 2018a. Organophosphate ester flame retardants in Nepalese soil: Spatial distribution, source apportionment and air-soil exchange assessment. Chemosphere 190, 114-123.

Yadav, I.C., Devi, N.L., Li, J., Zhang, G., Covaci, A., 2018b. Concentration and spatial distribution of organophosphate esters in the soil-sediment profile of Kathmandu Valley, Nepal: Implication for risk assessment. Science of the Total Environment 613-614, 502-512.

Yang, J., Li, Q., Li, Y., 2020a. Enhanced biodegradation/photodegradation of organophosphorus fire retardant using an integrated method of modified pharmacophore model with molecular dynamics and polarizable continuum model. Polymers 12, 1672.

Yang, Y., Xiao, Y., Chang, Y., Cui, Y., Klobučar, G., Li, M., 2018. Intestinal damage, neurotoxicity and biochemical responses caused by tris (2-chloroethyl) phosphate and tricresyl phosphate on earthworm. Ecotoxicology and Environmental Safety 158, 7886.

Yang, Y., Yin, H., Peng, H., Lu, G., Dang, Z., 2020b. Biodegradation of triphenyl phosphate using an efficient bacterial consortium GYY: Degradation characteristics, metabolic pathway and 16S rRNA genes analysis. Science of the Total Environment 713, 136598.

Zheng, C., Feng, S., Liu, P., Fries, E., Wang, Q., Shen, Z., Liu, H., Zhang, T., 2016. Sorption of organophosphate flame retardants on pahokee peat soil. Clean-Soil Air Water 44, 1163-1173.

Zhong, M., Wu, H., Mi, W., Li, F., Ji, C., Ebinghaus, R., Tang, J., Xie, Z., 2018. Occurrences and distribution characteristics of organophosphate ester flame retardants and plasticizers in the sediments of the Bohai and Yellow Seas, China. Science of the Total Environment 615, 1305-1311.

Zhou, X., Liang, Y., Ren, G., Zheng, K., Wu, Y., Zeng, X., Zhong, Y., Yu, Z., Peng, P., 2020. Biotransformation of tris (2-chloroethyl) phosphate (TCEP) in sediment microcosms and the adaptation of microbial communities to TCEP. Environmental Science \& Technology 54, 5489-5497.

Zhu, Y., Zhang, J., Liu, Y., Su, G., Zhu, L., Lin, D., 2019. Environmentally relevant concentrations of the flame retardant tris (1,3-dichloro-2-propyl) phosphate inhibit the growth and reproduction of earthworms in soil. Environmental Science \& Technology Letters 6, 277-282. 